\title{
Head Gesture Based Control of Wheelchair for a Paralysed Person in an Indoor Environment
}

\author{
Mohammed Ismail.B ${ }^{1}$, Syed Fouzan Ishaqui ${ }^{2}$, Abdul Karim Khan', Mohammed Abdul Quadeer ${ }^{2}$ \\ Sr. Assistant Professor, EED. Muffakham Jah College of Engineering \& Technology, Hyderabad, India ${ }^{1}$ \\ Student, Electronics \& Instrumentation Engineering, M.J.C.E.T, Hyderabad, India ${ }^{2}$
}

\begin{abstract}
This paper implements \& develops a wheel chair control for the physically challenged by employing head gesture recognition using MEMS technology. Tremendous advances have been made in the field of wheelchair technology; however, even these significant advances haven't been effective in helping unassisted quadriplegics to navigate the wheelchair. Here, a simple cost effective wheelchair is developed which can be controlled by simple head gestures for directions like left, right, front, and back. An accelerometer MEMS sensor is used to control the wheelchair through head gestures made by the impaired person and moves accordingly. This paper also presents the experimental results on the movement responses of the developed wheel chair with patients of varying weight.
\end{abstract}

Keywords: Micro-electromechanical systems (MEMS), wheelchair, quadriplegic, Gesture

\section{INTRODUCTION}

The number of people, who need to move around with the help of some artificial means, due to an illness or accident, is continuously increasing. This means of their locomotion has to be increasingly sophisticated \& cost effectively work in order to enhance their quality of life and cement their integration into their working world [1].

Paralysis is of two types:

1. Quadriplegia

2. Paraplegia

Quadriplegia is derived from two words: "Quadra" means four and "Plegia" stands for paralysis. When a person has spinal cord injury above the first thoracic vertebra, paralysis usually affects cervical spinal nerves resulting dis-functioning of the four limbs. Paraplegia is derived from two words: "Para" means two and "Plegia" stands for paralysis. The person affected with paraplegia normally has dis-functioning of both their legs. [2]

Today's technology is fast shifting towards automation which minimizes the need for human intervention. Those mechanization-cum-human operators with the aid of machinery require muscular work whereas automation greatly reduces the need for not only human sensory but also mental requirements as well. Thus, the present day automated systems have less manual operations, more flexibility, reliability and high accuracy. Due to this demand, every field prefers automated control systems. Especially in the field of electronics automated systems as they are giving reliable performance.

A survey on the adequacy of electric wheelchairs shows that, according to clinicians, $40 \%$ of patients have difficulties in steering and manoeuvring in daily life environments [3]. These scenarios include situations in which the manoeuvring space is limited, the approach to furniture or objects is tightly constrained and the necessity to pass through doorways requires precise control. The study also shows that conventional navigation with joysticks can cause fatigue during long operational periods. Clinicians pointed out that approximately $50 \%$ of the patients that are unable to control a powered wheelchair by conventional methods would benefit from automated navigation systems. In this context, intelligent wheelchairs are a mobility aid especially suitable for severely motor and/or cognitively impaired people that have difficulties in driving standard powered wheelchairs. [3]

\section{LiteratURE REVIEW}

Various methods have been proposed for allowing disabled persons, including a quadriplegic to control a motorized wheelchair.

There are proposed methodologies in recent times which involve various gestures like hand gesture, accelerometer \& voice controlled, EEG based system etc.

\section{A. Hand Gesture}

In this paper, they utilized the acceleration data to recognize the hand gestures and then transfer the gesture information which indicates certain motion commands into the wheelchair's smooth motions. It's a trial method to realize the natural interaction for the older and handicapped with the wheelchair through the hand gestures [4].

\section{B. Accelerometer and Voice Controlled}

This work describes a wheelchair for physically disabled people \& developed it using voice recognition kit and MEMS motion sensor. A user dependent voice recognition system had been integrated in the wheelchair. In this way they had obtained a wheelchair which can be driven using both motion and voice commands [5].

\section{EEG System}

This system proposes two control modes: Use of the gyroscope Emotive EPOC headset in order to detect head movements. Emotive EPOC headset is a device that measures EEG activity from 14 saline electrodes. These electrodes are arranged according to the 10/20 sys-tem, 
and their locations are AF3, F7, F3, FC5, T7, P7, O1, O2, P8, T8, FC6, F4, F8 and AF4.[6]

The Nav Chair Assistive Wheelchair Navigation System, The Nav Chair has application for the development and testing of shared control systems where a human and the machine share control of a system and the machine can automatically adapt to human behaviours. The Nav Chair shares vehicle control decisions with the wheelchair operator regarding obstacle avoidance, safe object approach, maintenance of a straight path, and other navigational issues, to reduce the motor and cognitive requirements for operating a power wheelchair.

Touch Screen Based Direction and Speed Control of Wheel Chair for Physically Challenged, this paper describes an intelligent motorized wheel chair for handicapped person using touch screen technology. It enables a disabled person to move around independently using a touch screen application which is interfaced with motors through micro-controller. When we want to change the direction, the touch screen sensor is modelled to direct the user to the required destination using direction keys on the screen and that values are given to micro-controller. Depending on the direction selected on the touch screen, micro-controller controls the wheel chair directions. The speed controller works by varying the average voltage sent to the motor. This is done by switching the motors supply on and off very quickly using PWM technique. [5]

All the above discussed systems are though sophisticated but they are either too complex in operation or complex in their circuitry and are not cost effective. Here our developed Wheel chair system is simple in operation, has good response \& reasonably affordable for most of the users.

\section{Block Diagram of Proposed System}

The system consists of major components like PIC Microcontroller 16F877A with 8K Flash memory, Sensor MEMs MMA 7260Q, Two DC Motors of 195rpm and 20V DC supply, Driver L293D, PWM Circuit, Reset Emergency circuit, ADC, Crystal oscillator, LED circuitry etc. as shown in Fig 1 .

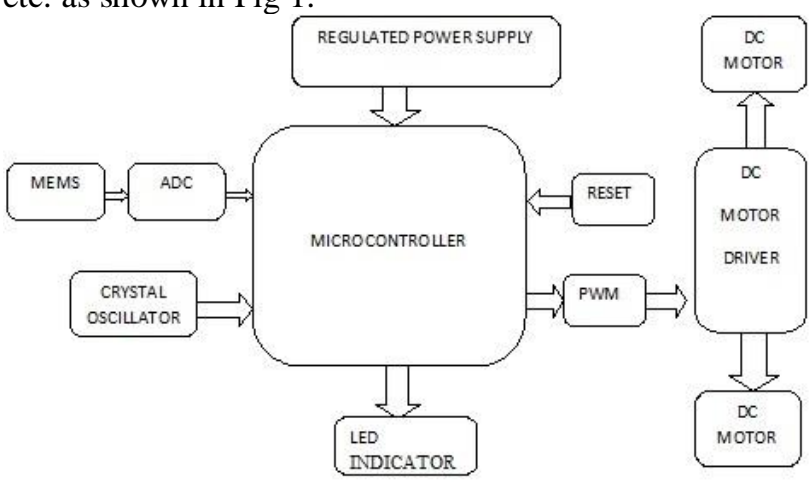

Fig. 1. Block Diagram of Setup

\section{IV.DESIGN DETAILS}

The schematic diagram of MEMS controlled intelligent wheel chair shows the interfacing section of each component with micro-controller PIC 16F877A with 8K X 14 word flash memory with operating frequency of DC to
$20 \mathrm{MHz}$ and MEMS sensor. The crystal oscillator, regulated power supply, LED's connected to microcontroller through resistors and motor driver circuitry is shown in Circuit Diagram fig 2.

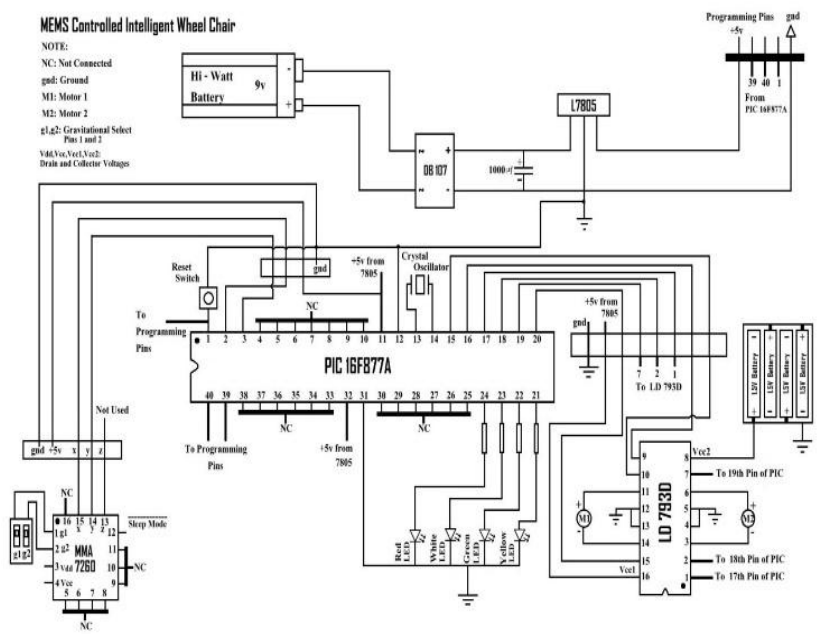

Fig. 2. Circuit Diagram of the Model

Different sections of the schematic diagram are explained in the following sections:

\section{A. Interfacing crystal oscillator and reset switch with} micro-controller

Crystal oscillator and reset button which are connected to the micro-controller. The two pins of oscillator are connected to the 13th and 14th pins of PIC microcontroller; the purpose of external crystal oscillator is to speed up the execution part of instructions per cycle and here the crystal oscillator has $20 \mathrm{MHz}$ frequency. The 1st pin of the micro-controller is referred to as MCLR i.e Master Clear Pin or Reset Input Pin is connected to reset button or power-on-reset. Reset option is kept for stopping the system at extreme conditions.

\section{B. Interfacing DC Motor Driver to Micro-controller}

The DC Motor is interfaced to the micro-controller through its driver IC L293D which is a 16 pin where as two D.C motors are connected to pins 3, 6, 11,14 of L293D and in turn the L293D is connected to Microcontroller Pins 4, 5, 6, 7(Port A) as shown in fig 3

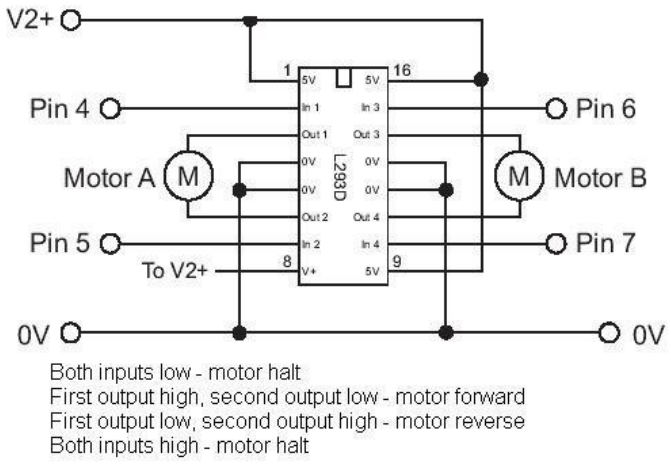

Fig. 3. Schematic Diagram of Motor Driver Circuit 


\section{MODEL Description}

The circuit model works on the wheels of chair switching relays \& motors with MEMS sensor placed on patients head. MEMS is used to send tilt signal to the wheelchair i.e., left or right or front or back. These tilt signals are passed to the controller as instructions. According to the program written for the controller, controller will give instructions to the wheelchair via relay. Here relay acts as a switching circuit. According to the relay operation wheelchair will move in that corresponding direction. If both the inputs to the Motor Driver are Low and high at the same time than the motor is in halt position. If the first output is high, Second output is low then DC Motor moves forward. If the first output is low, second output is high as shown in the circuit model figure 4 then DC Motor moves reverse.

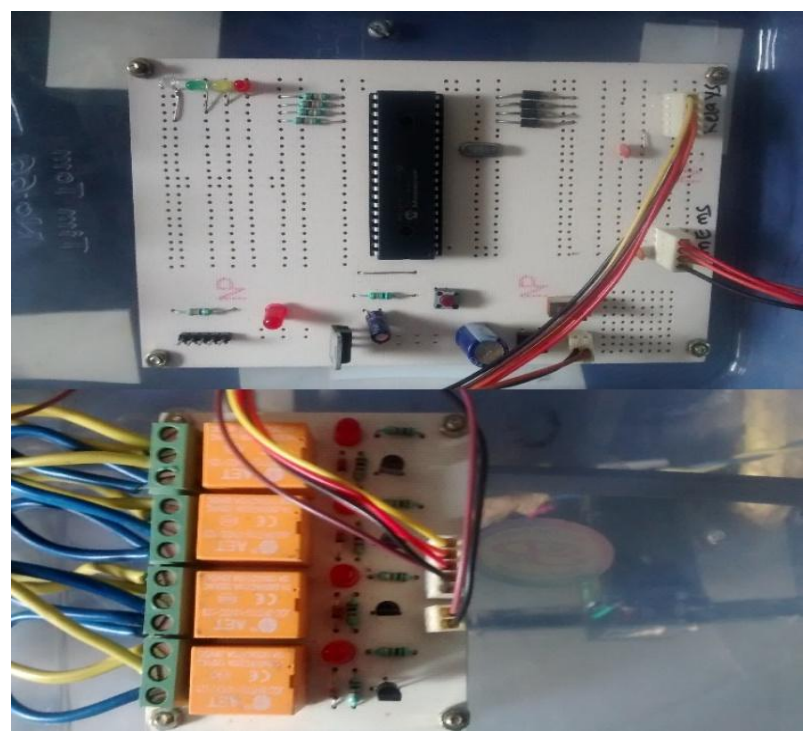

Fig. 4. Circuit of the working Model

\section{WORKING MODEL OF WHEELCHAIR}

Figure 5.shows the developed working model of Head Gesture based control Wheel chair which was tested under standard conditions and can be used in an indoor environment.

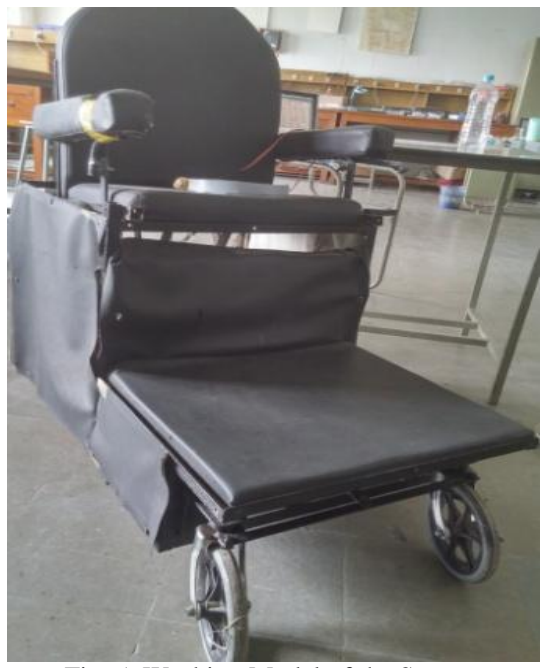

Fig. 5. Working Model of the System

\section{EXPERIMENTAL RESUlts \& Discussions}

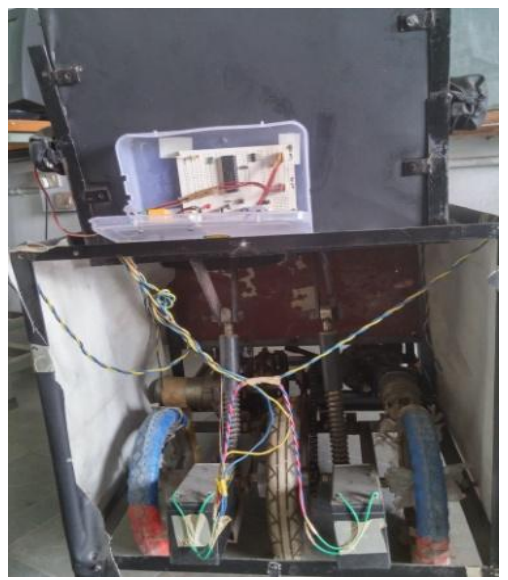

Fig. 6. Rear view of the wheel chair

The developed Wheelchair is tested for two conditions as discussed below:

TABLE I

Tabular column of Measured Values

\begin{tabular}{|c|c|c|c|c|}
\hline \multirow{2}{*}{ Direction } & \multirow{2}{*}{$\begin{array}{l}\text { Distanc } \\
\text { e (mts.) }\end{array}$} & No load & $30 \mathrm{~kg}$ load & $60 \mathrm{~kg}$ load \\
\hline & & Time (sec) & Time (sec) & Time (sec) \\
\hline \multirow{5}{*}{ Forward } & 0 & 0 & 0 & 0 \\
\hline & 1 & 4.5 & 5.05 & 5.6 \\
\hline & 2 & 7.8 & 12.6 & 16.8 \\
\hline & 3 & 12.6 & 21.6 & 29.5 \\
\hline & 4 & 17.8 & 28.6 & 39.5 \\
\hline \multirow{5}{*}{ Backward } & 0 & 0 & 0 & 0 \\
\hline & 1 & 4.5 & 6.5 & 8.5 \\
\hline & 2 & 8.8 & 14.5 & 19.3 \\
\hline & 3 & 13.6 & 20.9 & 28.8 \\
\hline & 4 & 17.8 & 28.5 & 38.5 \\
\hline \multirow{5}{*}{ Right } & 0 & 0 & 0 & 0 \\
\hline & 1 & 5.3 & 6.95 & 8.6 \\
\hline & 2 & 10.3 & 14.5 & 18.8 \\
\hline & 3 & 16.6 & 24.5 & 32.5 \\
\hline & 4 & 20.3 & 31.5 & 42 \\
\hline \multirow{5}{*}{ Left } & 0 & 0 & 0 & 0 \\
\hline & 1 & 5.3 & 6.95 & 8.6 \\
\hline & 2 & 10.3 & 14.5 & 18.8 \\
\hline & 3 & 16.6 & 24.5 & 32.5 \\
\hline & 4 & 20.3 & 31.5 & 42 \\
\hline
\end{tabular}

A. No load Condition

This includes wheelchair operation with no-load on it. The wheelchair is moved in forward, reverse, left \& right directions for a distance of 4 meters controlled by head gestures in the above mentioned directions for testing its behaviour. The response of the system is observed and tabulated as shown in Table 1.

\section{B. With load Condition}

Wheelchair is operated with a patient's weight of $30 \mathrm{~kg} \&$ $60 \mathrm{~kg}$ respectively on it. The wheelchair is observed to move in forward, reverse, left $\&$ right directions as per the 
head gestures for 4 meters of testing. Also the movement is tested for emergency stop if the Reset switch is pressed. The observations are tabulated \& plotted in terms of Distance Vs. Time Taken as shown in the Table 1 \& Graphs

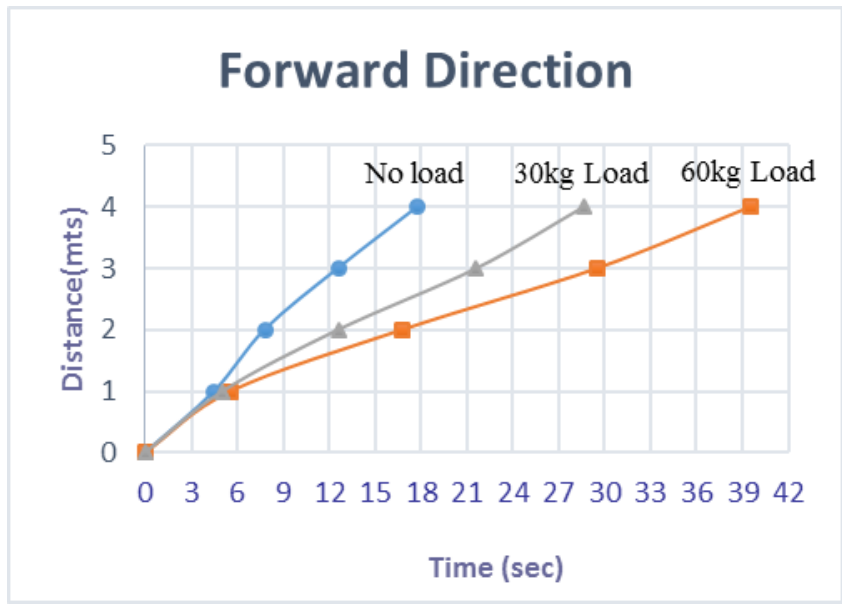

Fig. 7. Forward Direction. Distance vs. Time Graph

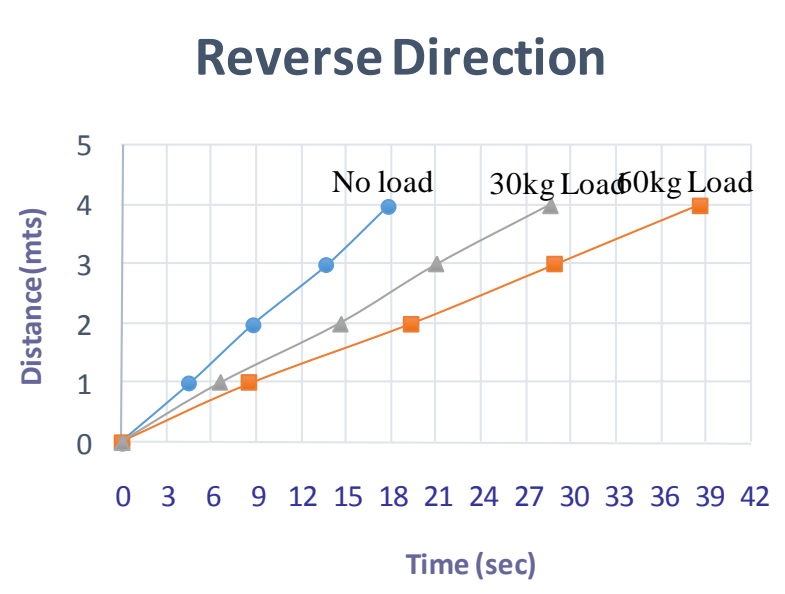

Fig. 8. Backward Direction: Distance vs. Time Graph

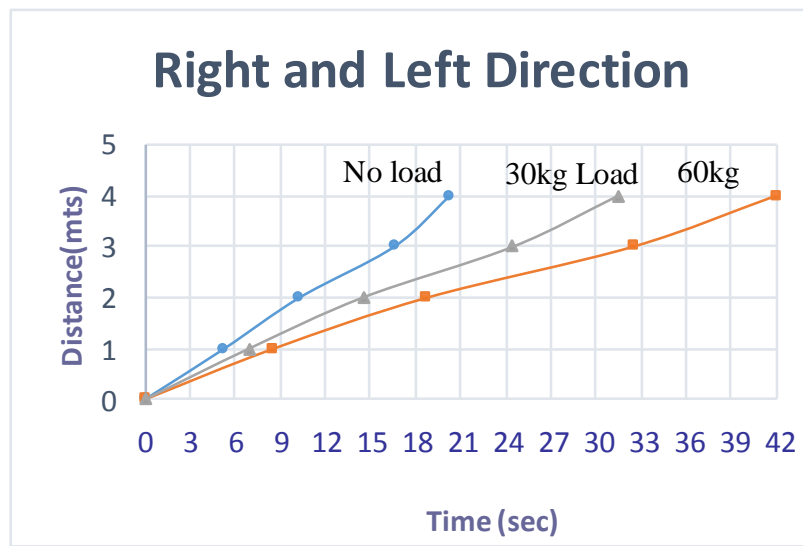

Fig. 9. Right and Left Direction: Distance vs. Time Graph

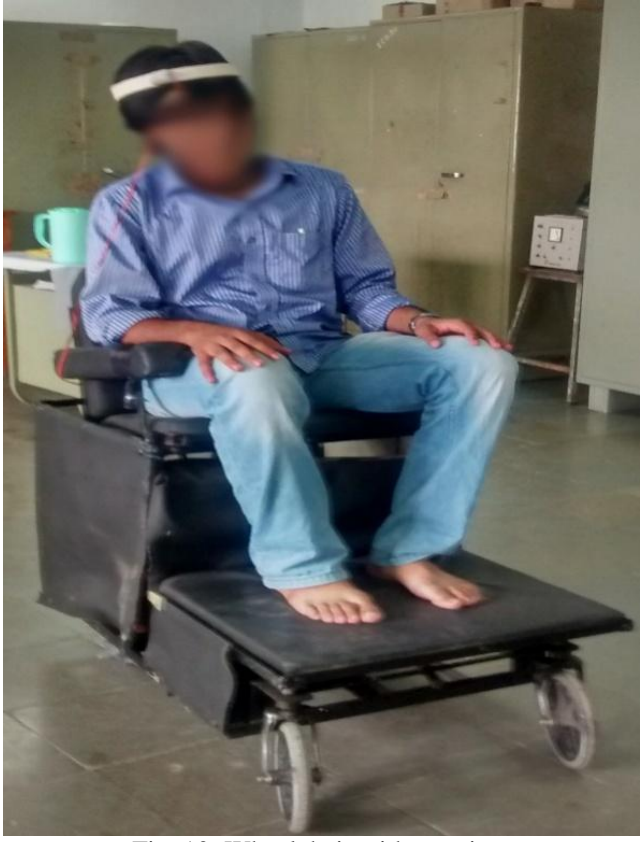

Fig. 10. Wheelchair with a patient

\section{Conclusion ANd Future Scope}

From the above obtained results, we conclude that the developed head gesture based control of wheel chair is tested and works satisfactorily in an indoor environment with minimum assistance to the person suffering with Quadriplegia or Paraplegia. It has a good response with MEMS activating the motors connected to the wheels of the chair.

The response and distance covered by wheelchair can be further improved if the gear system connected to motors are replaced by crank and pinion joint which has less friction and mechanical wear \& tear. In future we would work on this concept to improve the response and embed more sensors like proximity, ultrasonic, GPS to guide the impaired person in much more better way and use this wheel chair even under outdoor conditions.

\section{ACKNOWLEDGMENT}

With a deep sense of gratitude, we acknowledge the guidance, help \& active cooperation rendered by the following people whose guidance has sustained the effort and led to the successful completion of this work. Our sincere thanks to Dr. M. P. Soni, Head of Electrical Engineering Department for permitting us to use department labs of Integrated Circuits \& Microprocessors Micro-controllers to develop this system. We would like to convey our regards and thanks Dr. Basheer Ahmed, Advisor cum Director and Dr. Ashfaque Jafari Dean Academics, Muffakham Jah College of Engineering and Technology, Hyderabad for their support \& encouragement. We would also like to thank our technical staff Mr. Md. Faizuddin Lab Assistant EED for his assistance in developing this wheel chair.

\section{REFERENCES}

[1] S. Tameemsultana and N. Kali Saranya "Implementation of Head and Finger Movement Based Automatic Wheel Chair" Bonfring 
International Journal of Power Systems and Integrated Circuits, Vol. 1, Special Issue, December 2011

[2] Naveen Kumar, Rohini Deshpande, S. S. Manvi "Advanced Communication System for Quadri-plegics using PC and Accelerometer" International Journal of Emerging trends in Engineering and Development, ISSN 2249-6149 Issue1, Vol. 3(November-2011).

[3] Luis Montesano, Javier Minguez, Marta Diaz and Sonu Bhaskar "Towards an Intelligent Wheel-chair System for Cerebral Palsy Users" IEEE transactions on neural systems and rehabilitation engineering, vol. 18, no. 2, April 2010.

[4] Gowthaman.A, Ranjithkumar. R, Varunarajan. M V, Ganesh Babu.P U.G. Student "Wheel Chair Motion Control Based On Hand Gesture Recognition" International Journal of Innovative Research in Technology \& Science (IJIRTS) ISSN: 2321-1156.

[5] Rahul C. M., Nalini C. Iyer "accelerometer and voice controlled wheel chair" Proceedings of 4th SARC International Conference, 30th March-2014, Nagpur, India, and ISBN: 978-93-82702-70-2.

[6] Ericka Janet Rechy-Ramirez, Huosheng Hu and Klaus McDonaldMaier "Head movements based control of an intelligent wheelchair in an indoor environment" Proceedings of the 2012 IEEE, International Conference on Robotics and Biomometics, December 11-14,2012, Guang-zhou, China, 978-1-4673-2126-6/12.

[7] Shreedeep G., Somsubhra Mukherjee \& Soumya Chatterjee "Intelligent Gesture Controlled Wireless Wheelchair for the Physically Handicapped" Proceedings of Fifth IRAJ International Conference, 2013, India, ISBN: 978-93-82702-29-0 